\title{
Pneumonia mortality and healthcare utilization in young children in rural Bangladesh: a prospective verbal autopsy study
}

Farzana Ferdous ${ }^{1}$, Shahnawaz Ahmed ${ }^{2}$, Sumon Kumar Das²,3, Mohammod Jobayer Chisti ${ }^{2}$, Dilruba Nasrin ${ }^{4}$, Karen L. Kotloff5, Myron M. Levine ${ }^{5}$, James P. Nataro ${ }^{5,6}$, Enbo Ma ${ }^{7,9}$, Khitam Muhsen $^{8}$, Yukiko Wagatsuma9 ${ }^{9}$, Tahmeed Ahmed ${ }^{2}$ and Abu Syed Golam Faruque $2,10^{*}$

\begin{abstract}
Background: The present study aimed to examine the risk factors for death due to pneumonia in young children and healthcare behaviors of the guardians for children in rural Bangladesh. A prospective autopsy study was conducted among guardians of children aged 4 weeks to 59 months in Mirzapur, Bangladesh, from 2008 to 2012.

Results: Pneumonia was the primary cause of death, accounting for $26.4 \%(n=81)$ of all 307 deaths. Of the pneumonia deaths, 58\% ( $n=47)$ deaths occurred in younger infants (aged 4 weeks to $<6$ months) and 24.7\% $(n=20)$ in older infants (aged $6-11$ months). The median duration of illness before pneumonia death was 8 days (interquartile range $[\mathrm{IQR}] 3-20$ days). Prior to death, 91.4\% $(n=74)$ children with pneumonia sought treatment, and of those who sought treatment, $52.7 \%(n=39)$ sought treatment $\geq 2$ days after the onset of disease. Younger infants of 4 weeks to $<6$ months old were at 5.5-time (95\% confidence interval [CI] 2.5, 12.0) and older infants aged 6-11 months were at 3-time $(1.2,7.5)$ greater risk of dying from pneumonia than older children aged 12-59 months. Children with a prolonged duration of illness (2-10 days) prior to death were at more risk for death by pneumonia than those who died from other causes $(5.8[2.1,16.1])$. Children who died from pneumonia sought treatment 3.4-time more than children who died from other causes. Delayed treatment seeking ( $\geq 2$ days) behavior was 4.9-time more common in children who died from pneumonia than those who died from other causes. Children who died from pneumonia more often had access to care from multiple sources (5.7-time) than children who died from other causes.

Conclusions: Delay in seeking appropriate care and access to multiple sources for treatment are the underlying risk factors for pneumonia death in young children in Bangladesh. These results indicate the perplexity in guardians' decisions to secure appropriate treatment for children with pneumonia. Therefore, it further underscores the importance of focusing on mass media coverage that can outline the benefits of seeking care early in the progression of pneumonia and the potential negative consequences of seeking care late.
\end{abstract}

Keywords: Death, Infant, Health care, Health facilities, Pneumonia

\footnotetext{
* Correspondence: gfaruque@icddrb.org

${ }^{2}$ International Centre for Diarrhoeal Disease Research, Bangladesh (icddr,b),

Dhaka, Bangladesh

${ }^{10}$ Nutrition and Clinical Services Division, icddr,b, 68 Shaheed Tajuddin

Ahmed Sarani, Mohakhali, Dhaka 1212, Bangladesh

Full list of author information is available at the end of the article
}

(c) The Author(s). 2018 Open Access This article is distributed under the terms of the Creative Commons Attribution 4.0 International License (http://creativecommons.org/licenses/by/4.0/), which permits unrestricted use, distribution, and reproduction in any medium, provided you give appropriate credit to the original author(s) and the source, provide a link to the Creative Commons license, and indicate if changes were made. The Creative Commons Public Domain Dedication waiver (http://creativecommons.org/publicdomain/zero/1.0/) applies to the data made available in this article, unless otherwise stated. 


\section{Background}

Pneumonia is the leading cause of childhood death, accounting for $16 \%$ of 5.6 million deaths of children aged less than 5 years globally, more than $95 \%$ of which occur in developing countries [1]. In Bangladesh, pneumonia accounts for $15 \%$ of the 119,000 total deaths of children aged less than 5 years in 2015 [2].

Numerous pathogens can cause pneumonia. The respiratory syncytial virus, Streptococcus pneumoniae, and Haemophilus influenzae are the leading causes of childhood pneumonia, with the latter two being preventable through vaccination [3]. These vaccines are currently included in the immunization programs of numerous countries. The main risk factors for pediatric pneumonia include not being breastfed, undernutrition, indoor air pollution, household crowding, low birth weight, incomplete immunization, HIV, and pre-existing illnesses such as underlying heart disease [4, 5]. Muscle weakness, soft rib abnormalities, chest wall deformities, and impaired immune function may increase the severity of the disease [6-8]. Cyanosis [9], inability to feed, malnutrition $[9,10]$, prolonged duration of illness [11], altered mental state [10], and the presence of underlying chronic illness (such as heart disease) [12] are related with increased pneumonia-associated mortality in young children.

In 2010, 48 million deaths, 7 million of which were children, occurred in low- and middle-income countries (LMICs), and most of these deaths occurred without medical attention, at homes in rural areas [13, 14]. It is commonly believed that child mortality is higher in rural rather than in urban areas [15]. It has been also reported that the post-discharge mortality due to pneumonia is as high as in-hospital mortality even after receiving successful treatment at hospital [16]. Parents living in remote areas have a lack of adequate knowledge about clinical features of pneumonia and do not perceive the illness as serious or life-threatening [17]. Although Bangladesh has achieved $80 \%$ immunization coverage, only $22 \%$ of children receive postnatal checkups for immunizations and only $37 \%$ receive facility treatment for acute respiratory infections (ARI) [18]. However, 12\% of children do not receive any kind of treatment for ARI and a larger portion of ARI children do not receive facility treatment; their treatment forms do not yet reveal, where routine checkups have the potential to detect health problems and provide optimal health care [18]. Previous studies have highlighted prompt identification of symptoms or risk markers of pneumonia, and subsequent interventions may prevent complications and child deaths $[19,20]$. Even with these suggestions and solutions, there are some underlying risks that have not yet come to light that require understanding and consideration to reduce the risk of death by pneumonia in children. Verbal autopsy is a technique of growing importance used to estimate the distribution of the cause of death in populations lacking vital registries or other medical death certificates [21]. The technique focuses on the child or maternal deaths to elicit information on the signs, symptoms, and sequence of events during the final illness leading to death, which has been increasingly used in LMICs $[14,22]$. Therefore, this study aimed to examine the risk factors for deaths due to pneumonia in children less than 5 years of age in rural Bangladesh and describe patterns of healthcare utilization that preceded death.

\section{Methods}

\section{Study design and study population}

This prospective post-mortem verbal autopsy study was conducted from January 2008 to December 2012 within the framework of the Global Enteric Multicenter Study (GEMS). The present study comprised a secondary data analysis of risk factors of pneumonia mortality. The GEMS was conducted in four sites in sub-Saharan Africa (Gambia, Mali, Mozambique, and Kenya) and three sites in South Asia (India, Bangladesh, and Pakistan) and a rural sub-district (Mirzapur) which was the field site of the GEMS in Bangladesh [23, 24].

Mirzapur is a sub-district of the Tangail district located about $60 \mathrm{~km}$ north of Dhaka, the capital city of Bangladesh, and has a geographical area of $374 \mathrm{~km}^{2}$. The GEMS established a demographic surveillance system (DSS) in Mirzapur in January 2007 that covered eight out of 13 unions (the minimum administrative unit in rural Bangladesh). The DSS of GEMS regularly updated vital events including deaths in the study populations. In 2007, the mid-year population in the surveillance area was 238,463 . The crude birth and death rates were 20.7 and 5.6 per thousand populations, respectively. The total fertility rate was 2388 per thousand women aged 1549 years. The rate of neonatal mortality was 28.7 per thousand live births [25].

\section{Data collection-verbal autopsy}

Verbal autopsies were conducted in case of any deaths of a child aged less than 5 years in the DSS area. The DSS of Mirzapur collected information on demographic vital events three times a year (every 4 months). The total area was divided into 15 clusters; one data collector was responsible for each cluster, and each cluster was further divided into 80 blocks. Each data collector visited a block (40-50 households) each day and covered 80 blocks within 16 weeks. Additionally, four data collectors were assigned to collect the most recent information on pregnancy outcomes. The database was updated on a weekly basis, and a list of recent deaths of children less than 5 years old, including neonatal deaths, was generated to conduct verbal autopsies. Eventually, 
an overall list of total deaths of children aged less than 5 years was obtained and the information was cross-checked with the weekly list generated on all deaths to detect any mismatches and/or to complete information that was not provided in the weekly list.

The causes of death were classified according to International Classification of Disease 10th edition (ICD-10) codes [26]. Information on the causes of death was collected in a standardized manner from the medical chart or healthcare providers and, if available, death certificate. The caretaker, typically the mother, was interviewed by a trained research assistant using a World Health Organization (WHO) standard verbal autopsy questionnaire 3 or 4 weeks after the child's death [27]. Anonymous forms were reviewed by two clinicians independently to determine both the primary and antecedent causes of death of the child. In case of disagreement between the two clinicians, that was resolved through discussions with a third clinician. Detailed information on clinical signs and symptoms around the time of the child's death, as well as care-seeking practices, were obtained. The questionnaire included both closed and open-ended questions.

\section{Inclusion and exclusion criteria}

The present study only focused on data collected by the WHO verbal autopsy questionnaire for children aged 4 weeks to 59 months. Two different WHO verbal autopsy questionnaires were used for data collection from two different age groups of children (0-28 days and 4 weeks to 59 months). Given the difficulty in matching data obtained from these two age groups for analysis, data collected from another WHO verbal autopsy questionnaire for neonates aged $0-28$ days were excluded from present study analysis. We further excluded sepsis from the pneumonia risk factor analysis as severe cases of pneumonia can eventually lead to sepsis if not properly treated [28].

\section{Definitions}

According to WHO classification, pneumonia was defined as the "presence of a cough and/or respiratory difficulty with any of the following symptoms reported by caregivers, such as first breathing, chest wall indrawing, noisy breathing, or flaring of nostril" [9].

In this study, the term "infant" denotes a child who was 4 weeks to 11 months old with children aged 4 weeks to $<6$ months defined as "younger infants", and children 6 to 11 months old are defined as "older infants." The term "treatment" refers to any type of care including home remedies or other medical attention as reported by caregivers that were administered to the child with an illness that led to death. The term "indigenous healer" implies a person who is authorized by a particular culture or subculture to provide "indigenous treatment" even though he/she has not been so trained according to acceptable professional standards. The term, therefore, includes a whole range of individuals from religious shamans, witch doctors, and medicine men (homeopathic) within a community. The term "delay in treatment" represents a child who did not seek treatment within days of disease onset [29]. The term, therefore, considers a child with a delay of two and more days to seek treatment (median 1 day) for an illness that leads to death. "Malnutrition" denotes a child who had inadequate growth and thinness according to caregiver's perception.

\section{Statistical analysis}

Descriptive statistics were employed to describe the study sample and distribution of causes of death. The normality of the distribution was checked, and in case of a skewed distribution, median and interquartile range (IQR) values were given. Differences in the proportions of pneumonia death and other causes of death according to independent categorical variables were examined using the chi-square test. Crude and adjusted odds ratios (OR) and 95\% confidence intervals (CI) were calculated for each independent variable. Age of the children was categorized into three groups considering younger infants at higher risk of death: (i) 4 weeks to $<6$ months, (ii) 6 to 11 months, and (iii) 12 to 59 months (1259 months was not further categorized due to insufficient frequency). Based on the median number of days to begin treatment after the disease onset, children were classified into three groups: (i) delayed in seeking treatment for $\geq 2$ days, (ii) sought treatment within $0-1$ day, and (iii) did not seek any treatment. The duration of disease that leads to death was categorized into three groups denoting prolonged duration of illness ( $>10$ days) at a higher risk of death: (i) 11 days and more, (ii) 210 days, and (iii) $0-1$ day. Diagnosed medical conditions were classified into two groups based on presence or absence of any previous disease before the final illness that leads to death (yes/no). Before classifying diagnosed medical conditions into two groups, a cumulative frequency for all pre-existing diseases (congenital heart disease, malnutrition, kidney or liver diseases, cancer, asthma, and diarrhea) was estimated based on the presence or absence of the symptoms (yes/no). Symptoms noted during the final illness that leads to death were classified into two groups based on the presence or absence of any symptoms (yes/no). Before classification of symptoms into two groups, a cumulative frequency for all symptoms (diarrhea, vomiting, abdominal pain, no passage of stool, headache, presence of any mass, pain in neck, unconsciousness, convulsion, paralysis of lower limb, any change in urine flow, skin rash, red color of eyes, nasal bleeding, yellow color of urine, weight loss, thinness or wasting, and a change in hair color) was 
estimated based on the presence or absence of the symptoms (yes/no). Data from each source accessed for the treatment were collected separately. Responses from sources were merged into a separate variable, where government hospitals and government clinics were considered a single source, and as private hospitals, and private clinics were also considered as another single source of treatment.

Correlations between the independent variables were assessed using Spearman's correlation coefficient. Highly correlated variables such as variables measuring healthcare utilization (correlation coefficients $>0.70$ ) were analyzed in separate models to avoid multicollinearity among predictor variables. $P<0.10$ in bivariate analysis was set as an inclusion criterion of variables in the multivariable models along with known predisposing factors such as age at death, previous illness diagnosed of the child, and symptoms noted during illness (any symptoms reported by mother) before death. Several multivariable logistic regressions were performed to identify the risk factors for pneumonia (ICD-10-CM code J18.8) as the primary cause, whereas other causes of death were used as a comparison group. In model 1, we included the following variables: seeking treatment before death (yes or no), age at death, preexisting diagnosed illness, symptoms, and duration of illness before death (symptoms which were likely to lead to death). An additional two models included variables relating to healthcare utilization and the interval between receiving treatment and death due to pneumonia. In model 2 , all variables from model 1 were included except the variable "seeking treatment before death" which was replaced by another variable "sought care a number of days after onset of the disease." In model 3, similar variables of model 2 were included, except "sought care a number of days after onset of the disease" variable was replaced by a variable denoting the "number of sources accessed to seek treatment." $P<0.05$ was considered as statistically significant. IBM SPSS (version 23.0; New York, USA) was used for data entry and analysis. Chi-square for linear trend was calculated by EpiInfo software (version 7.2).

\section{Results}

From January 2008 to December 2012, a total of 24,561 children were born in the DSS area of the Mirzapur and $307(23.5 / 10,000)$ children aged 4 weeks to 59 months died during the study period. The cause-specific death rate for pneumonia $(6.2 / 10,000)$ did not change during the study period (Table 1). The mean age at death was 14.6 months [standard deviation (SD) 15.3] (median 9.0 months). Approximately, 40.7\% $(n=125)$ of all deaths occurred in younger infants ( 4 weeks to $<6$ months), $16.9 \%(n=52)$ occurred in older infants aged 6 to 11 months, and $42.3 \%(n=130)$ in children 12 to 59 months old. Overall, $26.4 \%(n=81)$ of all deaths were accounted for pneumonia. Other primary causes of deaths were sepsis (ICD-10-CM code A41.9) $(20.2 \% ; n=62$ ), drowning (18.9\%; $n=58)$ (ICD-10-CM code V92), diarrhea/dysentery $(9.4 \% ; n=29)$, congenital heart disease $(4.6 \% ; n=14)$, and illness suggestive of central nervous system $(\mathrm{CNS})$ disorders $(3.9 \% ; n=12)$ (Fig. 1).

To avoid overlapping clinical features, sepsis deaths (ICD-10-CM code A41.9) $(n=62)$ were excluded from the analysis of risk factors for death from pneumonia (Fig. 1). Of the children enrolled in the study $(n=245)$, $11.4 \%(n=28)$ had a previous diagnosis of heart disease; $11 \%(n=27)$ suffered from liver or kidney diseases, cancer, asthma, and diarrhea, etc.; and 4.9\% $(n=12)$ had malnutrition. During the final illness that leads to death, fever was noted among $40 \%(n=122)$ of the children and one third of them reported a cold and cough $(n=$ $84)$, difficulty in breathing $(n=98)$, and chest indrawing $(n=75)$. The median duration of illness before child death was 4 days (interquartile range [IQR] 1-15 days) for all children, and for pneumonia deaths, the median duration of illness before death was 8 days (IQR 320 days). The median duration before seeking treatment after disease onset was 1 day (IQR 0-60 days [median 1.5 days for pneumonia]). For $66.5 \%(n=163)$ of the children, treatment was sought before death, and of those who sought treatment, 67.5\% $(n=110)$ children were admitted to a hospital or health facility for treatment and, for $43.6 \%(n=71)$ children, treatment was

Table 1 Fertility and mortality indicators of rural Mirzapur DSS, 2008-2012

\begin{tabular}{llllllll}
\hline Indicators & 2008 & 2009 & 2010 & 2011 & 2012 & Overall & $p$ value \\
\hline Total population & 239,233 & 244,754 & 258,061 & 263,085 & 264,998 & 239,233 \\
Total under-5 population & 26,103 & 26,143 & 26,090 & 26,161 & 25,912 & 130,409 \\
Number of live births (CBR) & $5176(21.6)$ & $4844(19.8)$ & $5040(19.5)$ & $5156(19.6)$ & $4345(16.4)$ & $24,561(19.7)$ & 0.0 \\
Childhood deaths (4 weeks-59 months; CMR) & $62(23.8)$ & $62(23.7)$ & $61(23.4)$ & $59(22.6)$ & $63(24.3)$ & $307(23.5)$ & 0.9 \\
$\begin{array}{l}\text { Childhood pneumonia deaths (4 weeks-59 months; } \\
\text { CPMR) by under-5 population }\end{array}$ & $21(8.0)$ & $11(4.2)$ & $16(6.1)$ & $16(6.1)$ & $17(6.6)$ & $81(6.2)$ & 0.8 \\
\hline
\end{tabular}

Childhood death rate was measured as number of deaths per 10,000 total under-5 populations by each year period. Childhood pneumonia death rate was measured as number of pneumonia deaths per 10,000 under-5 population per year

CBR crude birth rate, CMR child mortality rate, CPMR childhood pneumonia mortality rate, DSS demographic surveillance system 


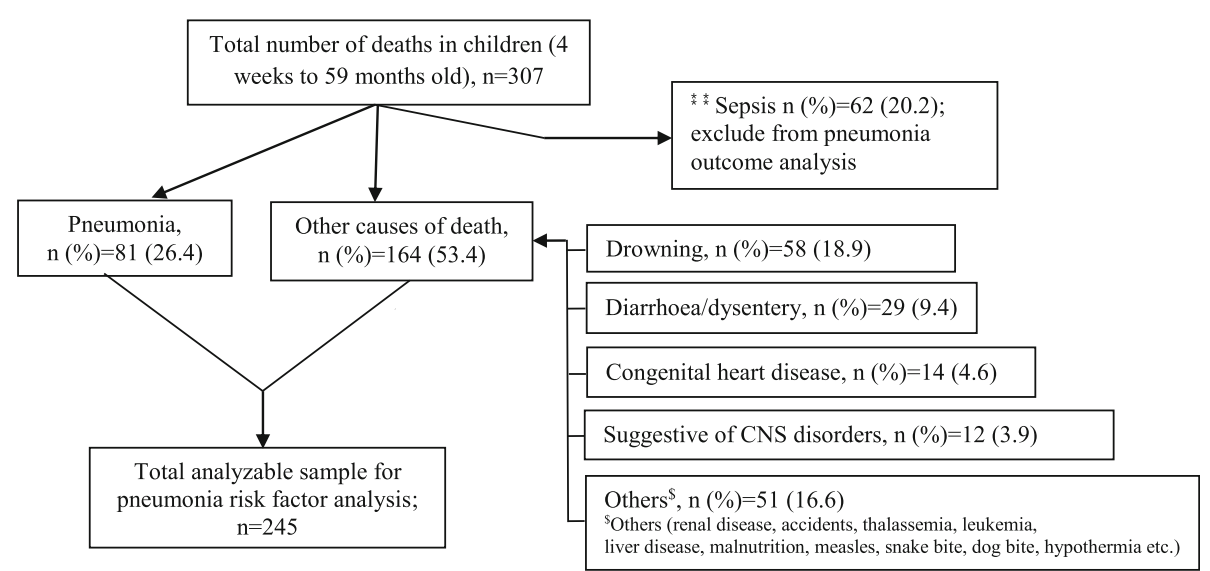

Fig. 1 Flowchart of primary causes of death of children aged 4 weeks-59 months. *** To avoid overlapping clinical features, sepsis deaths ( $n=62)$ were excluded from the analysis of risk factors for death from pneumonia

sought $\geq 2$ days after disease onset. Of those who sought treatment before death $(n=163)$, treatment was sought largely from private clinics/hospitals $(79.7 \% ; n=130)$, followed by government facilities $(28.2 \% ; n=46)$, pharmacies/drug stores $(28.2 \% ; n=46)$, indigenous healers $(16.6 \% ; n=27)$, and home treatment $(6.1 \% ; n=10)$ (note: guardians accessed multiple sources for their child's treatment; therefore, the figures do not add up to $100 \%$ ).

\section{Risk factors for pneumonia deaths}

Children aged 4 weeks to $<6$ months, and those aged 6 to 11 months, when compared with older children 12 to 59 months old, were at increased risk to die from pneumonia than children who died from other causes. Children who died from pneumonia were ill for a longer period before death than those who died from other causes (53.2 vs. $21.4 \%$ were ill for $2-10$ days, and 38 vs. $28.3 \%$ were ill for 11 days or more, respectively; $p<$ 0.05). Children who died from pneumonia received treatment for their illnesses more often than those who died from other causes. Children who died from pneumonia were more likely to seek treatment $\geq 2$ days after the onset of the illness than children who died from other causes (Table 2). Of the children with pneumonia who had visited three and more sources for treatment $(n=14), 71.4 \%(n=10)$ of them delayed in seeking treatment until $\geq 2$ days after the onset of disease and $75.0 \%$ $(n=11)$ of them had a duration of illness $\geq 11$ days before death. Of those children with pneumonia who visited two sources for treatment $(n=24), 41.7 \%(n=10)$ of them were delayed in seeking treatment $\geq 2$ days after onset of disease and $58.3 \% \quad(n=14)$ of them had a duration of illness of 2-10 days before death (data not shown in the table).

Highly significant correlations $(r>0.7, p<0.05)$ were found between three variables: seeking treatment, time elapsed (in days) between disease onset and seeking care, and the number of sources accessed for treatment $(r=$ $0.869,0.854$, and 0.764 , respectively) (Additional file 1 : Table S1). As a result, these variables were not included in the same multivariable model.

Multivariable model 1 showed that younger infants aged 4 weeks to $<6$ months were 5-time and older infants $6-$ 11 months old were 3-time at increased risk of death for pneumonia than the older children 12-59 months old. Children with pneumonia had a prolonged duration of illness (6-fold more), particularly with duration of illness of 2-10 days than children who died from other causes. Children with pneumonia were more likely to seek treatment (3-fold more) than children who died from other causes (Table 2). The present study performed additional analyses (multivariable analyses) to identify knowledge gaps between care-seeking behavior and deaths from pneumonia. Multivariable model 2 showed that, in addition to age and duration of illness, children with pneumonia, who delayed $\geq 2$ days from the onset of disease to seek care, were at more risk of death than the children who died from other causes. Multivariable model 3 showed that children with pneumonia were more likely to visit multiple sources ( 3 or more) to seek treatment than children who died due to other causes (Additional file 2: Table S2).

\section{Discussion}

We assessed the risk factors for death due to pneumonia in children aged less than 5 years old in rural Bangladesh. Pneumonia was the leading cause of death found in 81/307 of all deaths of children aged 4 weeks to 59 months. Children who died from pneumonia were predominantly infants, with those aged less than 6 months being particularly at a higher risk. The present study revealed some important findings relating to death 
Table 2 Correlates of pneumonia deaths compared to other causes of deaths in children aged 4 weeks to 59 months in rural Bangladesh $(n=245)$

\begin{tabular}{|c|c|c|c|c|}
\hline Variables & $\begin{array}{l}\text { Pneumonia, } \\
n=81(\%)\end{array}$ & $\begin{array}{l}\text { Other causes of death, } \\
n=164(\%)\end{array}$ & $\begin{array}{l}\text { Unadjusted OR } \\
(95 \% \mathrm{Cl})\end{array}$ & $\begin{array}{l}\text { Adjusted OR } \\
(95 \% \mathrm{Cl})^{* * *}\end{array}$ \\
\hline \multicolumn{5}{|l|}{ Age } \\
\hline 4 weeks -5 months & $47(58.0)$ & $38(23.2)$ & $8.9(4.4-18.0)^{*}$ & $5.5(2.5-12.0)^{*}$ \\
\hline 6-11 months & $20(24.7)$ & $25(15.2)$ & $5.1(2.6-13.0)^{*}$ & $3.0(1.2-7.5)^{*}$ \\
\hline $12-59$ months & $14(17.3)$ & $101(61.6)$ & 1 & 1 \\
\hline \multicolumn{5}{|l|}{ Sex } \\
\hline Male & $41(50.6)$ & $85(51.8)$ & $0.5(0.6-1.6)$ & NA \\
\hline Female & $40(49.4)$ & $79(48.2)$ & 1 & 1 \\
\hline \multicolumn{5}{|l|}{ History of medical condition (multiple frequency) } \\
\hline Heart disease & $14(17.3)$ & $14(8.5)$ & $2.2(1.0-5.0)^{*}$ & $1.4(0.6-3.0)$ \\
\hline Malnutrition & $5(6.2)$ & $7(4.3)$ & $1.5(0.5-4.8)$ & \\
\hline Others (liver disease, kidney disease, cancer, asthma, diarrhea, etc.) & $10(12.3)$ & $17(10.4)$ & $1.2(0.4-2.8)$ & \\
\hline None & $52(64.1)$ & $126(76.8)$ & 1 & 1 \\
\hline Small at birth $\left(<1\right.$ year), $n=131^{\dagger}$ & $21 / 67(31.3)$ & $16 / 64(25.0)$ & $1.4(0.6-3.0)$ & NA \\
\hline Premature $\left(<37\right.$ weeks) $(<1$ year $), n=131^{\dagger}$ & $15 / 67(22.4)$ & $4 / 64(6.3)$ & $4.3(1.4-13.9)^{*}$ & NA \\
\hline \multicolumn{5}{|l|}{ Symptoms noted during last illness (multiple frequency) } \\
\hline Fever & $44(54.3)$ & $54(32.3)$ & $2.5(1.4-4.3)^{*}$ & $0.9(0.4-2.2)$ \\
\hline Vomiting & $13(16.0)$ & $34(20.7)$ & $0.7(0.4-1.5)$ & \\
\hline Not passed stool & $5(6.2)$ & $22(13.4)$ & $0.4(0.2-1.2)$ & \\
\hline Unconscious & $6(7.4)$ & $15(9.1)$ & $0.8(0.3-2.1)$ & \\
\hline Convulsion & $9(11.1)$ & $17(10.4)$ & $1.1(0.5-2.5)$ & \\
\hline Others & $39(48.1)$ & $67(40.9)$ & $1.3(0.8-2.3)$ & \\
\hline None & $35(43.2)$ & $73(44.5)$ & 1 & 1 \\
\hline \multicolumn{5}{|l|}{ Duration of illness that leads to death (days), $n=238$} \\
\hline$\geq 11$ & $30(38.0)$ & $45(28.3)$ & $7.6(3.1-18.7)^{*}$ & $2.5(0.8-7.4)$ \\
\hline $2-10$ & $42(53.2)$ & $34(21.4)$ & $14.1(5.8-34.6)^{*}$ & $5.8(2.1-16.1)^{*}$ \\
\hline $0-1$ & $7(8.9)$ & $80(50.3)$ & 1 & 1 \\
\hline \multicolumn{5}{|l|}{ Sought care for illness that leads to death } \\
\hline Yes & $74(91.4)$ & $89(54.3)$ & $8.9(3.9-20.5)^{*}$ & $3.4(1.1-10.1)^{*}$ \\
\hline No & $7(8.6)$ & $75(45.7)$ & 1 & 1 \\
\hline \multicolumn{5}{|l|}{ Sought care a number of days after onset of the disease (days) } \\
\hline$\geq 2$ & $39(48.1)$ & $32(19.5)$ & $12.4(5.0-30.7)^{*}$ & NA \\
\hline $0-1$ & $35(43.2)$ & $57(34.8)$ & $7.0(2.9-16.7)^{*}$ & \\
\hline Did not seek treatment for illness & $7(8.6)$ & $75(45.7)$ & 1 & \\
\hline \multicolumn{5}{|l|}{ Admission to a hospital/health facility, $n=163 \neq$} \\
\hline Yes & $54(73.0)$ & $56(62.2)$ & $1.6(0.8-3.2)$ & NA \\
\hline No & $20(27.0)$ & $34(37.8)$ & 1 & \\
\hline \multicolumn{5}{|l|}{ Sources of treatment (multiple frequency), $n=163 \neq$} \\
\hline Home & $5(6.8)$ & $4(4.5)$ & $1.5(0.4-5.9)$ & NA \\
\hline Indigenous healer & $12(16.2)$ & $15(16.9)$ & $0.9(0.4-2.2)$ & \\
\hline Government clinic/hospital & $17(23.0)$ & $29(32.6)$ & $0.6(0.3-1.2)$ & \\
\hline Private clinic/hospital & $62(83.8)$ & $68(76.4)$ & $1.6(0.7-3.5)$ & \\
\hline Pharmacies/drug stores & $22(29.7)$ & $24(27.0)$ & $1.1(0.6-2.3)$ & \\
\hline
\end{tabular}

Number of sources accessed to seek treatment 
Table 2 Correlates of pneumonia deaths compared to other causes of deaths in children aged 4 weeks to 59 months in rural Bangladesh $(n=245)$ (Continued)

\begin{tabular}{|c|c|c|c|c|}
\hline Variables & $\begin{array}{l}\text { Pneumonia, } \\
n=81(\%)\end{array}$ & $\begin{array}{l}\text { Other causes of death, } \\
n=164(\%)\end{array}$ & $\begin{array}{l}\text { Unadjusted OR } \\
(95 \% \mathrm{Cl})\end{array}$ & $\begin{array}{l}\text { Adjusted OR } \\
(95 \% \mathrm{Cl})^{* * *}\end{array}$ \\
\hline$\geq 3$ & $14(17.3)$ & $15(9.1)$ & $10.0(3.5-29.0)^{*}$ & NA \\
\hline 2 & $24(29.6)$ & $30(18.3)$ & $8.6(3.3-22.0)^{*}$ & \\
\hline 1 & $36(44.4)$ & $44(26.8)$ & $8.8(3.6-21.4)^{*}$ & \\
\hline 0 (not sought treatment) & $7(8.6)$ & $75(45.7)$ & 1 & 1 \\
\hline \multicolumn{5}{|l|}{ Place of death } \\
\hline Hospital & $33(40.7)$ & $29(17.7)$ & $3.2(1.8-5.8)^{*}$ & NA \\
\hline Home & $48(59.3)$ & $135(82.3)$ & 1 & \\
\hline
\end{tabular}

Children who died from sepsis were exlcuded from the analysis

$O R$ odds ratio, $\mathrm{Cl}$ confidence interval, $\mathrm{NA}$ not applicable (those variables not included in the mutivariable model)

${ }^{*} p<0.05$

${ }^{\dagger}$ Only available sample, $n=131$

* Only available sample, $n=238$

¥Only available sample, $n=163$

***Model 1: dependent variable; causes of death $(1=$ pneumonia, $0=$ other causes); variables included in the model were age at death, previous diagnosed illness (used as binominal variable-yes or no), symptoms noted during final illness (used as binominal variable-yes or no), duration of illness that leads to death, and sought care for illness that leads to death

due to pneumonia in children and knowledge gaps in healthcare utilization by guardians of ill children in a rural community of Bangladesh. Firstly, only two thirds of these study children received treatment. Secondly, we noted that children who died from pneumonia had a prolonged duration of illness (median of 8 days) before death. Thirdly, a trend of delayed care seeking ( $\geq 2$ days) after the onset of disease was noted in children who died from pneumonia rather than other causes. Lastly, children who died from pneumonia sought treatment from multiple sources more often before death than children who died from other causes. Altogether, these findings suggest that children who died from pneumonia had a time window between onset of symptoms and death, in which appropriate treatment could have been delivered to reduce the risk of death. However, in most cases, appropriate action was not taken during this critical timing. To our knowledge, this is the first study that posits delay in seeking care and access to multiple sources for treatment, as key factors for delaying appropriate care for pneumonia in children.

In seeking treatment, one single child was witnessed to utilize a maximum of four sources, including treatment at home (home remedy). This clearly indicates the reasons for delayed care seeking behavior for illness, which may be associated with families' socioeconomic context and additional determining factors such as inadequate parental knowledge about the disease $[17,30]$. The present study findings are consistent in some respects with previous studies as well as a WHO report which revealed the reasons for delayed seeking care (median 7 days) are treatment of children at home by the informal sector or indigenous healers, and such practices were important key barriers to prompt treatment and reduction of unnecessary childhood deaths due to pneumonia [20,29]. In the present study, half of the children suffered at least 4 days due to the illness (for pneumonia, 8 days) and delayed at least 1 day (for pneumonia, 1.5 days) to seek treatment for the illness that leads to death. This lag time in between disease onset, treatment, and death of the children can be explained by the treatment sources those had been accessed by the guardians of ill children. Of those who sought treatment, one fourth $(28.2 \%$; $29.7 \%$ for pneumonia) of them attempted to seek treatment from pharmacies/drug stores, or $16.6 \%$ from indigenous healers $(16.2 \%$ for pneumonia), or $6.1 \%$ received remedies at home (6.8\% for pneumonia); additionally, $33.5 \%$ children never received any treatment for illnesses that lead to death (9\% for pneumonia), suggesting inappropriate healthcare utilization patterns by guardians of sick children in this rural community of Bangladesh. It could be possible that guardians of pneumonia children accessed many different facilities, and due to the inappropriate or inadequate quality of care, their children could not recover and, subsequently, died. The present WHO verbal autopsy questionnaire for 4 weeks to 59 months old children could not reveal much meaningful information such as treatment quality at the facility and the places where the child was taken first to seek treatment.

According to UNICEF 2016 report, children younger than 2 years were at higher risk of death from pneumonia [31]; however, in the present study, infants $(<1$ year old), especially less than 6 months old, were at a higher risk of death due to pneumonia than the older group. It has been reported that the immune system of children less than 6 months or 1 year old may be weakened if they are malnourished or if have not exclusively breastfed [32], though present study was unable to reveal those associations due to lack of those specific data. Former studies have indicated 
that guardians from rural as well as urban communities are unable to recognize the signs and symptoms of pneumonia, particularly the danger signs of pneumonia; thus, there is a delay in seeking care $[17,33]$. In the present study, children with pneumonia often were reported as having symptoms such as fever, cough, and common cold. Diagnosis based on difficulty in breathing, chest indrawing, and noisy breathing (grunting or wheezing) can be easily performed to identify cases of childhood pneumonia or to measure the severity of disease. However, the present study findings indicate that caregivers were found not to pay careful attention to these symptoms at the time critical for ensuring appropriate treatment. Like other developing countries, the severity of a disease was not given due attention in rural Bangladesh at the initial stage, and parents tried to seek treatment from drug stores or local unlicensed healthcare providers [17, 20, 34]. These practices may have also occurred to the present study children. The advanced stage of the disease characterized by high fever, cough, difficulty in breathing, and first breathing may have influenced family members in the present study to take their children to the health facility, yet it appears guardians did not access them at the appropriate time. It has been also observed that $40 \%$ of children with pneumonia died at the hospital; however, $74 \%$ of children who died from pneumonia were admitted to hospitals to receive treatment for their illnesses. This gap between death and admission of children with pneumonia has been explained by Chisti et al. who reported that even after a successful treatment at a hospital, post-discharge mortality has been reported to be as high as in-hospital mortality [16] which suggests the possible existence of persistent subclinical infection even after recovery or the survivor of premature microbiota in these children.

Previous studies have reported that inadequate education, lack of decision-making in the family, poor household socioeconomic status, longer distance from healthcare facility, and inadequate knowledge about disease severity are the key barriers to accessing health care [17, 34-36]. Moreover, overall factors such as quality of care, costs, geographic barrier, and existing social network influence families to rely on community-level healthcare providers for their care [37]. Access to qualified healthcare providers to receive care is often impeded due to a lack of household income and the location of the family in a remote area, which is often accentuated by physical geographic barriers or the lack of transportation to reach the health facility in a timely manner [17, 37, 38].

Guardians of children living in areas similar to this study should be knowledgeable about child health and the advance signs and symptoms of common morbidities and further be aware of the appropriate measures necessary for prevention and early treatment. Moreover, programs, such as community-based treatment of childhood pneumonia, should consider the referral of children with complications to larger facilities for better treatment. A previous study in Zambia suggested health education in the rural community can help community members recognize clinically severe pneumonia and understand the importance of early and appropriate care seeking [39]. This study also proposes training and retraining of personnel in different levels of health care with an emphasis on case definition and case management, where management includes preventive measures and appropriate means of referral. Such training should prioritize the use of available protocols for health workers who play a key role in maternal and child health clinics, especially in the rural settings. By promoting appropriate care for young children, the mortality rate of children less than 5 years old would be reduced in Bangladesh, especially in the case of infants.

Limitations of the study

In the present study, all the information was collected on the basis of recall method (usually 3 to 4 weeks after death); to overcome this recall bias, trained research assistants carefully interviewed respondents which were again reviewed by research physicians. It is often accepted that details pertaining to stressful events like death are not easily forgotten; thus, authors believe these recalls as likely to be accurate [40]. Additionally, verbal autopsy questionnaires that were developed by WHO have some generic limitations due to lacking information on parent's education, family income, the number of antenatal care (ANC) visits made by pregnant women, breastfeeding history, rate of breathing/minute, and the places where the first attempts to seek care were made. However, the likelihood of missing of data after conducting interviews was avoided by regular checks by the DSS personnel. Any mismatch or confusion on diagnosis was also avoided through quality training and reviewing of questionnaires by a group of trained and experienced physicians.

\section{Conclusions}

Pneumonia is the number one killer in children less than 5 years of age in rural Bangladesh based on post-mortem verbal autopsies, and deaths mostly occur in infants. This study addresses important knowledge gaps by identifying factors such as caregivers' delay in seeking appropriate care and access to multiple sources of treatment as the underlying risk factors for death due to pneumonia in young children in Bangladesh. The study findings suggest a time window between onset of symptoms and death for pneumonia, in which appropriate treatment can be delivered to reduce the risk of death. Prompt identification and treatment of less severe cases of pneumonia at the beginning may prevent their progression to severe pneumonia and death. A mass media program at the community level aimed at educating caregivers about the simple clinical features of pneumonia and intelligent utilization of health services might reduce childhood pneumonia deaths in 
Bangladesh. The government of Bangladesh should consider setting priorities and formulating appropriate strategies to educate the population to ensure improved healthcare behaviors which may address the basic need to improve health care in a resource-constraint setting like Bangladesh. Such an effort would achieve better results if both the public and the private sectors are involved in service delivery in rural Bangladesh.

\section{Additional files}

Additional file 1: Table S1. Correlation between pneumonia and co-variates. (DOC $42 \mathrm{~kb}$ )

Additional file 2: Table S2. Correlates of pneumonia deaths compared to other causes of deaths in children aged 4 weeks to 59 months in rural Bangladesh. (DOC $56 \mathrm{~kb}$ )

\section{Abbreviations}

ANC: Antenatal care; Cl: Confidence intervals; CNS: Central nervous system; DSS: Demographic surveillance systems; GEMS: Global Enteric Multicenter Study; ICD-10: International Classification of Disease 10th edition codes; IQR: Interquartile range; LMICs: Low- and middle-income countries; OR: Odds ratios; SD: Standard deviation; UNICEF: The United Nations Children's Fund; WHO: World Health Organization

\section{Acknowledgements}

The icddr,b acknowledges with gratitude the commitment of The Bill \& Melinda Gates Foundation (BMGF) to its research efforts. We acknowledge the following donors for providing unrestricted support to icddr,b's effort and advancement to its strategic plan: the Government of the People's Republic of Bangladesh and Canada (Global Affairs, Canada-GAC), Sweden (Sida), and the United Kingdom (DFID). The authors would lastly like to thank Joshua Gallagher (Department of Health Services Research, University of Tsukuba) for his contribution in English language editing to finalize the paper.

\section{Funding}

This research protocol was funded by The Bill \& Melinda Gates Foundation (BMGF), grant number GR-00505 (OPP 1033572).

\section{Availability of data and materials}

The dataset and protocol of the current study are accessible through the corresponding author and can be accessed on reasonable request.

\section{Authors' contributions}

FF, EM, KM, and ASGF performed, analyzed, and interpreted the present study and played the major roles in writing the manuscript. FF, SKD, KM, EM, YW, and ASGF analyzed and interpreted all the data produced and contributed to the writing of the manuscript. All authors read and approved the final manuscript.

\section{Ethics approval and consent to participate}

The GEMS was approved by the Institutional Review Board (IRB) of the University of Maryland (UMB) and the Research Review Committee (RRC) and Ethical Review Committee (ERC) of the International Centre for Diarrhoeal Disease Research, Bangladesh (icddr,b). The post-mortem verbal autopsy was part of GEMS which was also approved by the IRB of UMB and RRC and ERC of icddr,b. Verbal consent was obtained from parents, and the ERC was satisfied with the voluntary participation, maintenance of the rights of the participants, and confidential handling of personal information by the research personnel and approved this consenting procedure.

\section{Competing interests}

The authors declare that they have no competing interests.

\section{Publisher's Note}

Springer Nature remains neutral with regard to jurisdictional claims in published maps and institutional affiliations.

\section{Author details}

${ }^{1}$ Graduate School of Comprehensive Human Sciences, University of Tsukuba, Tsukuba, Japan. ${ }^{2}$ International Centre for Diarrhoeal Disease Research, Bangladesh (icddr,b), Dhaka, Bangladesh. ${ }^{3}$ School of Public Health, The University of Queensland, Brisbane, Australia. ${ }^{4}$ Center for Vaccine Development and Department of Medicine, University of Maryland School of Medicine, Baltimore, MD, USA. ${ }^{5}$ Center for Vaccine Development, Department of Pediatrics and Medicine, University of Maryland School of Medicine, Baltimore, MD, USA. 'Department of Pediatrics, University of Virginia School of Medicine, Charlottesville, VA, USA. 'Health Promotion Center, Fukushima Medical University, Fukushima, Japan. ${ }^{8}$ Department of Epidemiology and Prevention Medicine, School of Public Health, Sackler Faculty of Medicine, Tel Aviv, Israel. ${ }^{9}$ Department of Clinical Trial and Clinical Epidemiology, Faculty of Medicine, University of Tsukuba, Tsukuba, Japan.

${ }^{10}$ Nutrition and Clinical Services Division, icddr,b, 68 Shaheed Tajuddin Ahmed Sarani, Mohakhali, Dhaka 1212, Bangladesh.

Received: 19 February 2018 Accepted: 20 May 2018

Published online: 25 May 2018

\section{References}

1. UNICEF, Levels \& trends in child mortality. Report 2017. New York: the United Nations Children's fund; 2017.

2. UNICEF, Committing to child survival. A promise renewed. Progress report 2015. In: New York: the United Nations Children's fund; 2015.

3. Leung DT, Chisti MJ, Pavia AT. Prevention and control of childhood pneumonia and diarrhea. Pediatr Clin N Am. 2016;63:67-79.

4. Rudan I, Boschi-Pinto C, Biloglav Z, Mulholland K, Campbell H. Epidemiology and etiology of childhood pneumonia. Bull World Health Organ. 2008;86:408-16.

5. Jackson S, Mathews KH, Pulanic D, et al. Risk factors for severe acute lower respiratory infections in children: a systematic review and meta-analysis. Croat Med J. 2013:54:110-21.

6. Spooner V, Barker J, Tulloch S, et al. Clinical signs and risk factors associated with pneumonia in children admitted to Goroka hospital, Papua New Guinea. J Trop Pediatr. 1989;35:295-300.

7. Suwanjutha S, Ruangkanchanasetr S, Chantarojanasiri T, Hotrakitya S. Risk factors associated with morbidity and mortality of pneumonia in Thai children under 5 years. Southeast Asian J Trop Med Public Health. 1994:25:60-6. 7825027.

8. Banajeh SM, al-Sunbali NN, al-Sanahani SH. Clinical characteristics and outcome of children aged under 5 years hospitalized with severe pneumonia in Yemen. Ann Trop Paediatr. 1997;17:321-6.

9. WHO, Pocket book of hospital care for children. Guidelines for the management of common childhood illnesses. In: Geneva: WHO; 2013.

10. Subhi R, Adamson M, Campbell H, et al. The prevalence of hypoxaemia among ill children in developing countries: a systematic review. Lancet Infect Dis. 2009;9:219-27.

11. Shann F, Barker J, Poore P. Clinical signs that predict death in children with severe pneumonia. Pediatr Infect Dis J. 1989:8:852-5.

12. Nascimento-Carvalho CM, Rocha H, Santos-Jesus R, Benguigui Y. Childhood pneumonia: clinical aspects associated with hospitalization or death. Braz J Infect Dis. 2002:6:22-8.

13. Jha P. Reliable direct measurement of causes of death in low- and middleincome countries. BMC Med. 2014:12:19.

14. Leitao J, Desai N, Aleksandrowicz L, et al. Comparison of physician-certified verbal autopsy with computer-coded verbal autopsy for cause of death assignment in hospitalized patients in low- and middle-income countries: systematic review. BMC Med. 2014;12:22.

15. Susuman AS. Child mortality rate in Ethiopia. Iran J Public Health. 2012; 41:9-19. 23113145.

16. Chisti MJ, Salam MA, Bardhan PK, et al. Treatment failure and mortality amongst children with severe acute malnutrition presenting with cough or respiratory difficulty and radiological pneumonia. PLoS One. 2015;10:e0140327.

17. Ferdous F, Dil Farzana F, Ahmed S, et al. Mothers' perception and healthcare seeking behavior of pneumonia children in rural Bangladesh. ISRN family med. 2014;2014:690315. https://doi.org/10.1155/2014/690315. 
18. Sayem AM, Nury AT, Hossain MD. Achieving the millennium development goal for under-five mortality in Bangladesh: current status and lessons for issues and challenges for further improvements. J Health Popul Nutr. 2011;29:92-102.

19. Graham SM, English M, Hazir T, Enarson P, Duke T. Challenges to improving case management of childhood pneumonia at health facilities in resourcelimited settings. Bull World Health Organ. 2008;86:349-55.

20. WHO/UNICE, WHO/UNICEF joint statement: management of pneumonia in community settings. 2004.

21. Sibai AM, Fletcher A, Hills M, Campbell O. Non-communicable disease mortality rates using the verbal autopsy in a cohort of middle aged and older populations in Beirut during wartime, 1983-93. J Epidemiol Community Health. 2001;55:271-6.

22. Hill K, Lopez AD, Shibuya K, Jha P. Monitoring of Vital E. Interim measures for meeting needs for health sector data: births, deaths, and causes of death. Lancet. 2007;370:1726-35.

23. Kotloff KL, Blackwelder WC, Nasrin D, et al. The global enteric multicenter study (GEMS) of diarrheal disease in infants and young children in developing countries: epidemiologic and clinical methods of the case/ control study. Clin Infect Dis. 2012;55(Suppl 4):S232-45.

24. Levine MM, Kotloff KL, Breiman RF, Preface ZAK. Am J Trop Med Hyg. 2013;

25. Darmstadt GL, Choi Y, Arifeen SE, et al. Evaluation of a cluster-randomized controlled trial of a package of community-based maternal and newborn interventions in Mirzapur, Bangladesh. PLoS One. 2010;5:e9696.

26. Engmann C, Jehan I, Ditekemena J, et al. An alternative strategy for perinatal verbal autopsy coding: single versus multiple coders. Tropical Med Int Health. 2011;16:18-29.

27. Perry HB, Ross AG, Fernand F. Assessing the causes of under-five mortality in the Albert Schweitzer hospital service area of rural Haiti. Rev Panam Salud Publica. 2005;18:178-86.

28. Florescu DF, Kalil AC. The complex link between influenza and severe sepsis. Virulence. 2014;5:137-42.

29. Kallander K, Hildenwall H, Waiswa P, Galiwango E, Peterson S, Pariyo G. Delayed care seeking for fatal pneumonia in children aged under five years in Uganda: a case-series study. Bull World Health Organ. 2008;86:332-8.

30. Chisti MJ, Duke T, Robertson CF, et al. Co-morbidity: exploring the clinical overlap between pneumonia and diarrhoea in a hospital in Dhaka, Bangladesh. Ann Trop Paediatr. 2011;31:311-9.

31. UNICEF, One is too many: ending child deaths from pneumonia and diarrhea. New York: UNICEF; 2016

32. Lamberti LM, Zakarija-Grkovic I, Fischer Walker CL, et al. Breastfeeding for reducing the risk of pneumonia morbidity and mortality in children under two: a systematic literature review and meta-analysis. BMC Public Health. 2013;13(Suppl 3):S18.

33. Hildenwall H, Nantanda R, Tumwine JK, et al. Care-seeking in the development of severe community acquired pneumonia in Ugandan children. Ann Trop Paediatr. 2009;29:281-9.

34. Das SK, Nasrin D, Ahmed S, et al. Health care-seeking behavior for childhood diarrhea in Mirzapur, rural Bangladesh. Am J Trop Med Hyg. 2013:89:62-8.

35. Ferdous F, Das SK, Ahmed S, et al. The impact of socio-economic conditions and clinical characteristics on improving childhood care seeking behaviors for families living far from the health facility. Science Journal of Public Health. 2013;1:69-76. https://doi.org/10.11648/.j.sph.20130102.14.

36. Sonego M, Pellegrin MC, Becker G, Lazzerini M. Risk factors for mortality from acute lower respiratory infections (alri) in children under five years of age in low and middle-income countries: a systematic review and metaanalysis of observational studies. PLoS One. 2015;10:e0116380.

37. Sack DA. Achieving the millennium development goals for health and nutrition in Bangladesh: key issues and interventions-an introduction. J Health Popul Nutr. 2008;26:253-60. 18831222

38. Shah R, Mullany LC, Darmstadt GL, et al. Determinants and pattern of care seeking for preterm newborns in a rural Bangladeshi cohort. BMC Health Serv Res. 2014;14:417.

39. Stekelenburg J, Kashumba E, Wolffers I. Factors contributing to high mortality due to pneumonia among under-fives in Kalabo district, Zambia. Tropical Med Int Health. 2002;7:886-93.

40. Lacy JW, Stark CEL. The neuroscience of memory: implications for the courtroom. Nat Rev Neurosci. 2013;14:649-58.

\section{Ready to submit your research? Choose BMC and benefit from:}

- fast, convenient online submission

- thorough peer review by experienced researchers in your field

- rapid publication on acceptance

- support for research data, including large and complex data types

- gold Open Access which fosters wider collaboration and increased citations

- maximum visibility for your research: over $100 \mathrm{M}$ website views per year

At BMC, research is always in progress.

Learn more biomedcentral.com/submissions 